\title{
Global Residue Harmonic Balance Method for a System of Strongly Nonlinear Oscillator
}

\author{
Huaxiong Chen (i) and Wei Liu (iD) \\ School of Mathematics and Statistics, Zhoukou Normal University, Zhoukou 466000, China \\ Correspondence should be addressed to Huaxiong Chen; chxecnu@163.com
}

Received 2 December 2020; Revised 23 July 2021; Accepted 23 August 2021; Published 9 September 2021

Academic Editor: Muhammad Arif

Copyright (C) 2021 Huaxiong Chen and Wei Liu. This is an open access article distributed under the Creative Commons Attribution License, which permits unrestricted use, distribution, and reproduction in any medium, provided the original work is properly cited.

In this paper, the global residue harmonic balance method is applied to obtain the approximate periodic solution and frequency for a well-known system of strongly nonlinear oscillator in engineering. This method can improve accuracy by considering all the residual errors in deriving each order approximation. With this procedure, the expressions of the higher-order approximate solution and corresponding frequency for the considered system can be determined easily. The comparison of the obtained results with previously existing and corresponding exact solutions shows the high accuracy and efficiency of the method.

\section{Introduction}

It is well known that the most models can be described by nonlinear differential equations. It is difficult to obtain exact solutions for these nonlinear problems. Thus, to find analytically approximate solutions to these nonlinear problems has been the desire of many researchers for a long time.

The classical perturbation methods [1-3] for solving nonlinear equations are limited to the weakly nonlinear systems. To overcome this shortcoming, many improved or effective methods applicable to the strongly nonlinear systems have been developed. These methods include the homotopy perturbation method (HPM) [4-6], the energy balance method [7], the harmonic balance method [8], the variational iteration method [9], and the Lattice Boltzmann method [10-12]. Also, many researchers have applied these methods to study the models in many different fields. By applying the homotopy analysis method (HAM), Easif et al. [13] obtained the approximate solutions of the variable coefficient variant boussinesq system, Vakilzadeh et al. [14] investigated the dynamic pull-in instability of a suspended microchannel resonator, and Suharsono et al. [15] studied the solution of the reverse flow reactor model. Abed [16] studied the Kuramoto-Sivashinsky equation by the new iterative method. Talarposhti et al. [17] analysed the solution to micropolar nanofluid between two parallel plates in a rotating system by using AGM. Gerdroodbary [18] simulated the magnetohydrodynamic (MHD) flow of a nanofluid inside an inclined enclosure with a sinusoidal wall by the CVFEM method. Khalouta and Kadem [19] investigated the solution of a class of nonlinear fractional partial differential equations by using the reduced differential transform method. Ramos [20] solved Bratu's and Troesch's problems by the hybrid block method. Peiravi and Alinejad [21] studied a 3D Newtonian droplet impact by the volume of fluid (VOF) method.

Among these strongly nonlinear methods, the global residue harmonic balance method (GRHBM) is an effective and analytical one. The GRHBM was the first presented by Ju and Xue [22] to obtain the periodic solutions of the jerk equation. By introducing a parameter $p$ and viewing it as a small parameter, the GRHBM can be independent on the large parameter and can give the higher-order analytical approximation. And, to improve the accuracy, the residual errors are considered in the approximations of each order. Thus, the GRHBM has found applications in various nonlinear problems [23-26].

In this paper, we study a system of strongly nonlinear oscillators in engineering, namely, the large amplitude free vibration of a restrained uniform beam carrying an 
intermediate lumped mass. This system was first presented by Hamdan and Shabaneh [27] and then has been analysed by Ganji et al. [28] by applying AFF and HPM. However, the higher-order analytical approximate solution for this system has not been obtained. To obtain high-order and more accurate results, this system is revisited in this paper by using the GRHBM. The higher-order approximate solution and corresponding frequency have been obtained. Comparison of the results obtained by using GRHBM with those obtained by exact solutions and other methods shows that the GRHBM can be very accurate, effective, and straightforward. It also can be extended to other nonlinear oscillators in engineering and physics.

\section{System Discussion}

A schematic of the beam under research is given in Figure 1. Here, we give a brief description of this beam. For more details, one can refer to [27].

The beam is considered to be uniform of length $l$ and unit mass $m$, hinged at the base to a rotational spring $K_{r}$ and carried a lumped mass $M$ at an arbitrary point $s=d$ along the beam span. $\zeta=s / l$ is a dimensionless arc length.

According to [27], the Lagrangian of this beam should be

$$
L=\left(\frac{m l}{2}\right) \int_{0}^{1}\left\{[1+\mu \delta(\zeta-\eta)]\left(\dot{x}^{2}+\dot{y}^{2}\right)-\left(\frac{E I \lambda^{4}}{2 m}\right)\left[y^{\prime \prime 2}+\left(\lambda y^{\prime} y^{\prime \prime}\right)^{2}+\lambda^{4} y^{\prime 4} y^{\prime \prime 2}\right]\right\} \mathrm{d} \zeta
$$

where the primes and dots denote the derivatives with respect to $\xi$ and $t$, respectively, $\eta=d / l$ is the relative position of $M, \mu=M / m l$ is a mass ratio parameter, $\delta(\zeta-\eta)$ is Dirac's function, $\lambda=1 / l$, and $E I$ is the modulus of flexural rigidity.

Assume $x$ and $y$ satisfy the constraint relation as follows:

$$
\left(1+\lambda x^{\prime}\right)^{2}+\left(\lambda y^{\prime}\right)^{2}=1 .
$$

Equation (2) can be rewritten as

$$
1+\lambda x^{\prime}=\left[1-\left(\lambda y^{\prime}\right)^{2}\right]^{1 / 2} .
$$

Assume $\left(\lambda y^{\prime}\right)^{2} \ll 1$ and $y(\zeta, t)$ have a form as follows:

$$
y(\zeta, t)=\phi(\zeta) u(t)
$$

where $\phi(\zeta)$ is an eigenfunction.

Expanding the right side of equation (3) to the fifthorder term, then equation (3) yields

$$
x=-\frac{1}{2} \int_{0}^{\zeta}\left(\lambda y^{\prime 2}+\frac{1}{4} \lambda^{3} y^{\prime 4}\right) \mathrm{d} x .
$$

Combining with equations (5) and (4), equation (1) can become

$$
\begin{array}{r}
L=\left(\frac{m l}{2}\right)\left\{\alpha_{1} \dot{u}^{2}+\alpha_{3} \lambda^{2} u^{2} \dot{u}^{2}+\alpha_{4} \lambda^{2} u^{2} \dot{u}^{2}+\alpha_{5} \lambda^{4} u^{4} \dot{u}^{2}+\alpha_{6} \lambda^{4} u^{4} \dot{u}^{2}\right. \\
\left.-\left(\frac{E I \lambda^{4}}{m}\right)\left(\alpha_{2} u^{2}+\alpha_{7} \lambda^{2} u^{4}+\alpha_{8} \lambda^{4} u^{6}\right)\right\},
\end{array}
$$

where $\alpha_{1}, \alpha_{2}, \ldots, \alpha_{8}$ are functions of $\zeta$, which describe the mode shapes.

Applying the Euler-Lagrange equation

$$
\frac{\mathrm{d}}{\mathrm{d} t}\left(\frac{\partial L}{\partial \dot{u}}\right)-\frac{\partial L}{\partial u}=0,
$$

to equation (6), one obtains the following problem:

$$
\ddot{q}+q+\varepsilon_{1} q^{2} \ddot{q}+\varepsilon_{1} q \dot{q}^{2}+\varepsilon_{2} q^{4} \ddot{q}+2 \varepsilon_{2} q^{3} \dot{q}^{2}+\varepsilon_{3} q^{3}+\varepsilon_{4} q^{5}=0,
$$

with

$$
\begin{aligned}
& q(0)=A, \\
& \dot{q}(0)=0,
\end{aligned}
$$

which $\varepsilon_{1}, \ldots, \varepsilon_{4}$ are functions of $\alpha_{1}, \ldots, \alpha_{8}$.

\section{Review of the Global Residue Harmonic Balance Method}

Consider the systems as follows:

$$
\begin{array}{cl}
\Phi(\ddot{q}, \dot{q}, q) & =0, \\
q(0) & =A, \\
\dot{q}(0) & =0,
\end{array}
$$

where $\dot{q}=\mathrm{d} q / \mathrm{d} t, A$ is the amplitude of the system, and $\Phi(\ddot{q}, \dot{q}, q)$ is a nonlinear function satisfying

$$
\Phi(-\ddot{q},-\dot{q},-q)=-\Phi(\ddot{q}, \dot{q}, q) .
$$

By introducing $\tau=\omega t$, equation (10) becomes

$$
\begin{aligned}
\Phi\left(\omega^{2} q^{\prime \prime}, \omega q^{\prime}, q\right) & =0, \\
q(0) & =A, \\
q^{\prime}(0) & =0,
\end{aligned}
$$

where $q^{\prime}=\mathrm{d} q / \mathrm{d} \tau$ and $\omega$ is an frequency.

Let the periodic solution of equation (12) exist, and it can be expressed by such a set of functions as follows:

$$
\{\cos (2 m-1) \tau \mid m=1,2,3, \ldots\} .
$$

According to equation (13), the initial approximation solution satisfying the initial conditions should be 


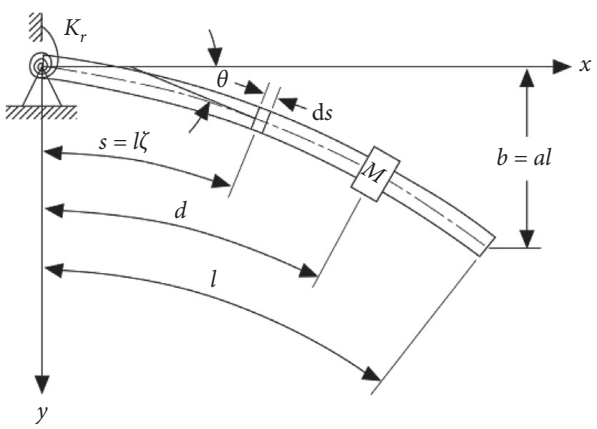

Figure 1: A sketch of the beam system under research.

$$
\begin{aligned}
q_{0}(\tau) & =A \cos \tau, \\
\tau & =\omega_{0} t,
\end{aligned}
$$

where $\omega_{0}$ can be determined later.

Substituting equations (14) into (12), the initial residual is

$$
R_{0}(\tau)=\Phi\left(\omega_{0}^{2} q_{0}^{\prime \prime}, \omega_{0} q_{0}^{\prime}, q_{0}\right)
$$

If $R_{0}(\tau)=0$, then $q_{0}(\tau)$ turns into the exact solution of equation (10). Generally, such case will not appear in the nonlinear systems.

Eliminating the secular terms in equation (15), through which, $\omega_{0}$ can be determined. Then, the zeroth-order approximation solution is obtained of the form as follows:

$$
\begin{aligned}
q_{0}(\tau) & =A \cos \tau, \\
\tau & =\omega_{0} t,
\end{aligned}
$$

with the initial residual as follows:

$$
R_{0}(\tau)=\Phi\left(\omega_{0}^{2} q_{0}^{\prime \prime}, \omega_{0} q_{0}^{\prime}, q_{0}\right)
$$

Next, we introduce a parameter $p \in[0,1]$, denote $q(\tau)$ and $\omega$ as $q(\tau, p)$ and $\omega(p)$, and expand $q(\tau, p)$ and $\omega(p)$ as follows:

$$
\begin{aligned}
q(\tau, p) & =q_{(k-1)}(\tau, p)+p^{k} q_{k}(\tau)+\cdots \\
\omega^{2}(p) & =\omega_{(k-1)}^{2}(p)+p^{k} \omega_{k}+\cdots
\end{aligned}
$$

in which

$$
\begin{aligned}
q_{(k-1)}(\tau, p) & =q_{0}(\tau)+p q_{1}(\tau)+\cdots+p^{k-1} q_{k-1}(\tau), \\
\omega_{(k-1)}^{2}(p) & =\omega_{0}^{2}+p \omega_{1}+\cdots+p^{k-1} \omega_{k-1} .
\end{aligned}
$$

Obviously, $q(t, 0)$ is the zeroth-order approximation and $q(t, 1)$ is the required approximate solution of equation (12).
Generally, the higher-order approximation to the solution can be provided by equation (18). For instance, the firstorder approximation can be shown as

$$
\begin{aligned}
q(\tau) & =q_{0}(\tau)+p q_{1}(\tau), \\
\omega^{2} & =\omega_{0}^{2}+p \omega_{1} .
\end{aligned}
$$

Inserting equations (20) into (12) and equating the coefficients of $p$, we can get

$$
\begin{aligned}
F_{1}\left(\tau, \omega_{1}, q_{1}(\tau)\right) & =\left.\frac{\mathrm{d}}{\mathrm{d} p}\left(\Phi\left(\omega^{2}(p) q^{\prime \prime}(\tau, p), \omega(p) q^{\prime}(\tau, p), q(\tau, p)\right)\right)\right|_{p=0} \\
& =\left(\omega_{1} \frac{\partial}{\partial\left(\omega^{2}\right)}+q_{1}^{\prime \prime} \frac{\partial}{\partial q^{\prime \prime}}+q_{1}^{\prime} \frac{\partial}{\partial q^{\prime}}+q_{1} \frac{\partial}{\partial q}\right) \Phi_{0},
\end{aligned}
$$

where $\partial \Phi_{0} / \partial q$ denotes that $\partial \Phi / \partial q$ is to be evaluated at the zeroth-order approximation.

By taking equation (13) into account, let

$$
q_{1}(\tau)=a_{3,1}(\cos \tau-\cos 3 \tau),
$$

and substitute it into equation (21), and we consider the following equation:

$$
R_{1}(\tau)=F_{1}\left(\tau, \omega_{1}, q_{1}(\tau)\right)+R_{0}(\tau),
$$

where $R_{0}(\tau)$ has been introduced into equation (23) to improve the accuracy.

The right hand side of equation (23) should not contain the terms $\cos \tau$ and $\cos 3 \tau$. Letting their coefficients be zeros, one could obtain two linear equations, then the two unknowns $\omega_{1}$ and $a_{3,1}$ can be determined. Till now, the firstorder approximation can be obtained as

$$
\begin{aligned}
q_{(1)}(\tau) & =q_{0}(\tau)+q_{1}(\tau), \\
\omega_{(1)}^{2} & =\omega_{0}^{2}+\omega_{1}, \\
\tau & =\omega_{(1)} t,
\end{aligned}
$$

with the residual

$$
R_{1}(\tau)=\Phi\left(\omega_{(1)}^{2} q_{(1)}^{\prime \prime}, \omega_{(1)} q_{(1)}^{\prime}, q_{(1)}\right)
$$

For the $k$-th-order approximation, we may assume

$$
q_{k}(\tau)=\sum_{i=1}^{k} a_{2 i+1, k}[\cos \tau-\cos (2 i+1) \tau], \quad k=2,3, \ldots, .
$$

To determine $a_{2 i+1, k},(i=2, \ldots, k)$ and $\omega_{k}$, substituting equations (18) into (12) and collecting the coefficients of $p$, one should get

$$
\begin{aligned}
F_{k}\left(\tau, \omega_{k}, q_{k}(\tau)\right) & =\left.\frac{1}{k !} \frac{\mathrm{d}^{k}}{\mathrm{~d} p^{k}}\left(\Phi\left(\omega^{2}(p) q^{\prime \prime}(\tau, p), \omega(p) q^{\prime}(\tau, p), q(\tau, p)\right)\right)\right|_{p=0}, \\
R_{k-1}(\tau) & =\Phi\left(\omega_{(k-1)}^{2}(1) q_{(k-1)}^{\prime \prime}(\tau, 1), \omega_{(k-1)}(1) q_{(k-1)}^{\prime}(\tau, 1), q_{(k-1)}(\tau, 1)\right) .
\end{aligned}
$$


Considering the equation

$$
R_{k}(\tau)=F_{k}\left(\tau, \omega_{k}, q_{k}(\tau)\right)+R_{k-1}(\tau),
$$

eliminating the coefficients of $\cos \tau, \cos 3 \tau, \ldots$ and $\cos (2 k+1) \tau$ in equation (28), then the unknowns $a_{3, k}, a_{5, k}, \ldots, a_{2 k+1, k}$ and $\omega_{k}$ can be obtained. Thus, the $k$-thorder approximation should be obtained as

$$
\begin{gathered}
q_{(k)}(\tau)=q_{(k-1)}(\tau, 1)+q_{k}(\tau), \\
\omega_{(k)}^{2}=\omega_{(k-1)}^{2}(1)+\omega_{k} .
\end{gathered}
$$

\section{Solution Procedure}

In this section, the GRHBM is applied to solve system (8) and (9). With $\tau=\omega t$, system (8) and (9) becomes

$$
\omega^{2}\left(q^{\prime \prime}+\varepsilon_{1} q^{2} q^{\prime \prime}+\varepsilon_{1} q q^{\prime 2}+\varepsilon_{2} q^{4} q^{\prime \prime}+2 \varepsilon_{2} q^{3} q^{\prime 2}\right)+q+\varepsilon_{3} q^{3}+\varepsilon_{4} q^{5}=0
$$

and

$$
\begin{aligned}
& q(0)=A, \\
& q^{\prime}(0)=0,
\end{aligned}
$$

where $q^{\prime}=\mathrm{d} q / \mathrm{d} \tau$.

4.1. Zeroth-Order Approximation. Firstly, the initial approximation can be written as

$$
\begin{aligned}
q_{0}(\tau) & =A \cos \tau, \\
\tau & =\omega_{0} t .
\end{aligned}
$$

Substituting equation (32) into (30) yields

$$
R_{0}(\tau)=F_{01} \cos \tau+F_{03} \cos 3 \tau+F_{05} \cos 5 \tau,
$$

in which

$$
\begin{aligned}
& F_{01}=\frac{8 A-8 \omega_{0}^{2} A-4 \omega_{0}^{2} \varepsilon_{1} A^{3}-3 \omega_{0}^{2} \varepsilon_{2} A^{5}+6 \varepsilon_{3} A^{3}+5 \varepsilon_{4} A^{5}}{8}, \\
& F_{03}=\frac{4 \varepsilon_{3} A^{3}+5 \varepsilon_{4} A^{5}-8 \omega_{0}^{2} \varepsilon_{1} A^{3}-7 \omega_{0}^{2} \varepsilon_{2} A^{5}}{16} \\
& F_{05}=\frac{\varepsilon_{4} A^{5}-3 \omega_{0}^{2} \varepsilon_{2} A^{5}}{16}
\end{aligned}
$$

Solving $F_{01}=0$ gives

$$
\omega_{0}=\sqrt{\frac{8+6 \varepsilon_{3} A^{2}+5 \varepsilon_{4} A^{4}}{8+4 \varepsilon_{1} A^{2}+3 \varepsilon_{2} A^{4}}},
$$

which is the same as the result by Ganji et al. [28]. Then, we obtain the zeroth-order approximation (32) and the initial residual error as follows:

$$
R_{0}(\tau)=F_{03} \cos 3 \tau+F_{05} \cos 5 \tau .
$$

4.2. First-Order Approximation. Substituting equations (20) and (22) into equation (30) and considering the coefficient of $p$, we have

$$
F_{1}\left(\tau, \omega_{1}\right)=\frac{F_{11} \cos \tau+F_{13} \cos 3 \tau+F_{15} \cos 5 \tau+F_{17} \cos 7 \tau}{16},
$$

in which

$$
\begin{aligned}
F_{11}= & 16 a_{3,1}-16 a_{3,1} \omega_{0}^{2}-16 A \omega_{1}-8 A^{3} \omega_{1} \varepsilon_{1}+5 A^{4} a_{3,1} \omega_{0}^{2} \varepsilon_{2}-6 A^{5} \omega_{1} \varepsilon_{2}+24 A^{2} a_{3,1} \varepsilon_{3}+25 A^{4} a_{3,1} \varepsilon_{4}, \\
F_{13}= & 144 a_{3,1} \omega_{0}^{2}-16 a_{3,1}+56 A^{2} a_{3,1} \omega_{0}^{2} \varepsilon_{1}-8 A^{3} \omega_{1} \varepsilon_{1}+31 A^{4} a_{3,1} \omega_{0}^{2} \varepsilon_{2}-7 A^{5} \omega_{1} \varepsilon_{2} \\
& -12 A^{2} a_{3,1} \varepsilon_{3}-5 A^{4} a_{3,1} \varepsilon_{4} \\
F_{15}= & 72 A^{2} a_{3,1} \omega_{0}^{2} \varepsilon_{1}+61 A^{4} a_{3,1} \omega_{0}^{2} \varepsilon_{2}-3 A^{5} \omega_{1} \varepsilon_{2}-12 A^{2} a_{3,1} \varepsilon_{3}-15 A^{4} a_{3,1} \varepsilon_{4} \\
F_{17}= & 31 A^{4} a_{3,1} \omega_{0}^{2} \varepsilon_{2}-5 A^{4} a_{3,1} \varepsilon_{4} .
\end{aligned}
$$

According to equation (23), we have

Eliminating the $\cos \tau$ and $\cos 3 \tau$ in equation (39) yields

$$
R_{1}(\tau)=F_{1}\left(\tau, \omega_{1}\right)+R_{0}(\tau) .
$$




$$
\begin{aligned}
0= & a_{3,1}-a_{3,1} \omega_{0}^{2}-A \omega_{1}+\frac{\varepsilon_{1} \omega_{1} A^{3}}{2}+\frac{5 a_{3,1} \varepsilon_{2} \omega_{0}^{2} A^{4}}{16}-\frac{3 \varepsilon_{2} \omega_{1} A^{5}}{8}+\frac{3 a_{3,1} \varepsilon_{3} A^{2}}{2}+\frac{25 a_{3,1} \varepsilon_{4} A^{4}}{16}, \\
0= & -a_{3,1}+9 a_{3,1} \omega_{0}^{2}-\frac{\varepsilon_{1} \omega_{0}^{2} A^{3}}{2}+\frac{7 a_{3,1} \varepsilon_{1} \omega_{0}^{2} A^{2}}{2}-\frac{\varepsilon_{1} \omega_{1} A^{3}}{2}-\frac{7 \varepsilon_{2} \omega_{0}^{2} A^{5}}{16}+\frac{31 a_{3,1} \varepsilon_{2} \omega_{0}^{2} A^{4}}{16} \\
& -\frac{7 \varepsilon_{2} \omega_{1} A^{5}}{16}+\frac{\varepsilon_{3} A^{3}}{4}-\frac{3 a_{3,1} \varepsilon_{3} A^{2}}{4}+\frac{5 \varepsilon_{4} A^{5}}{16}-\frac{5 a_{3,1} \varepsilon_{4} A^{4}}{16} .
\end{aligned}
$$

Solving equations (40) and (41) gives

$$
\begin{aligned}
& a_{3,1}=\frac{2 A^{3}\left(8+4 \varepsilon_{1} A^{2}+3 \varepsilon_{2} A^{4}\right) H}{2304 \omega_{0}^{2}-256 A^{2} \varepsilon_{1}+2176 A^{2} \omega_{0}^{2} \varepsilon_{1}-208 A^{4} \varepsilon_{2}-256+F+G}, \\
& \omega_{1}=\frac{A^{2}\left(16-16 \omega_{0}^{2}+5 \varepsilon_{2} \omega_{0}^{2} A^{4}+24 \varepsilon_{3} A^{2}+25 \varepsilon_{4} A^{4}\right) H}{2304 \omega_{0}^{2}-256 A^{2} \varepsilon_{1}+2176 A^{2} \omega_{0}^{2} \varepsilon_{1}-208 A^{4} \varepsilon_{2}-256+F+G},
\end{aligned}
$$

in which

$$
\begin{aligned}
H & =8 \varepsilon_{1} \omega_{0}^{2}+7 \varepsilon_{2} \omega_{0}^{2} A^{2}-4 \varepsilon_{3}-5 \varepsilon_{4} A^{2}, \\
G & =448 A^{4} \omega_{0}^{2} \varepsilon_{1}^{2}-240 A^{6} \varepsilon_{2} \varepsilon_{3}-192 A^{2} \varepsilon_{3}-240 A^{6} \varepsilon_{1} \varepsilon_{4}-205 A^{8} \varepsilon_{2} \varepsilon_{4}, \\
F & =1472 A^{4} \omega_{0}^{2} \varepsilon_{2}+544 A^{6} \omega_{0}^{2} \varepsilon_{1} \varepsilon_{2}+151 A^{8} \omega_{0}^{2} \varepsilon_{2}^{2}-80 A^{4} \varepsilon_{4}-288 A^{4} \varepsilon_{1} \varepsilon_{3} .
\end{aligned}
$$

Thus, we obtain the first-order approximation as follows:

$$
\begin{aligned}
\omega_{(1)} & =\sqrt{\omega_{0}^{2}+\omega_{1}}, \\
q_{(1)}(t) & =\left(A+a_{3,1}\right) \cos \left(\omega_{(1)} t\right)-a_{3,1} \cos \left(3 \omega_{(1)} t\right),
\end{aligned}
$$

where $\omega_{0}, \omega_{1}$, and $a_{3,1}$ are given in equations (35), (42), and (43).

With the procedure going on, the higher-order approximation can be obtained similarly.

4.3. Results and Discussion. To illustrate and verify the efficiency and accuracy of the presented method, the comparison of the analytical approximation obtained by the presented method with the exact solutions and other methods is discussed in this section. Here, the numerical results from fourth-order Runge-Kutta method are viewed as the exact solutions.

For this system, the parameters $\varepsilon_{i}(i=1,2,3,4)$ describe the mode shapes and can be calculated by the values of base stiffness $S$, mass ratio $\mu$, and position $\eta$. Here, the zeroth- and first-order approximate frequencies and their relative errors (RE) by the different values of $\varepsilon_{1} \sim \varepsilon_{4}$ and $A$ are shown in Table 1. From Table 1, it can be seen that the zeroth-order approximations of frequencies the same as the first-order results by HPM and AFF have a good adjustment with the exact ones with the maximal relative error 0.79887 , and the relative errors of the first-order approximate frequencies are much less than the zero-order. It indicates that the first-order approximate frequencies can be more closer to the exact frequencies than the zero-order ones, and the GRHBM is more efficient and more accurate for solving strongly nonlinear problems.

To further illustrate and verify the accuracy of the presenting method, we select some parameter values as follows:

$$
\begin{aligned}
& A=0.3, \\
& \varepsilon_{1}=8.205578, \\
& \varepsilon_{2}=3.145368, \\
& \varepsilon_{3}=0.272313, \\
& \varepsilon_{4}=0.133708, \\
& A=0.4, \\
& \varepsilon_{1}=5.074636, \\
& \varepsilon_{2}=1.971962, \\
& \varepsilon_{3}=0.281418, \\
& \varepsilon_{4}=0.149677, \\
& A=0.4, \\
& \varepsilon_{1}=4.233389, \\
& \varepsilon_{2}=1.657464, \\
& \varepsilon_{3}=0.318201, \\
& \varepsilon_{4}=0.173313, \\
& A=0.5, \\
& \varepsilon_{1}=1.642033, \\
& \varepsilon_{2}=0.913055, \\
& \varepsilon_{3}=0.313561, \\
& \varepsilon_{4}=0.204297,
\end{aligned}
$$

Figures 2-5 provide the comparisons of the current analytical solutions to the exact solutions. From Figures 2-5, 
TABle 1: Comparison of frequency corresponding to various parameters of this system.

\begin{tabular}{|c|c|c|c|c|c|c|c|c|}
\hline Mode & $A$ & $\varepsilon_{1}$ & $\varepsilon_{2}$ & $\varepsilon_{3}$ & $\varepsilon_{4}$ & $\omega_{0}=\omega^{(1)}[28](\mathrm{RE})$ & $\omega_{(1)}(\mathrm{RE})$ & $\omega_{e x}$ \\
\hline 1 & 1.0 & 0.326845 & 0.129579 & 0.232598 & 0.087584 & $1.00705(0.30664)$ & $1.010180(0.00297)$ & 1.01015 \\
\hline 2 & 0.5 & 1.642033 & 0.913055 & 0.313561 & 0.204297 & $0.93255(0.40947)$ & $0.935268(0.11982)$ & 0.93639 \\
\hline 3 & 0.2 & 4.051486 & 1.665232 & 0.281418 & 0.149677 & $0.96546(0.12144)$ & $0.965852(0.08152)$ & 0.96664 \\
\hline 4 & 0.3 & 8.205578 & 3.145368 & 0.272313 & 0.133708 & $0.85970(0.52752)$ & $0.864408(0.01712)$ & 0.86426 \\
\hline 1 & 1.2 & 0.303844 & 0.115076 & 0.232598 & 0.087584 & $1.02135(0.67990)$ & $1.027492(0.08246)$ & 1.02834 \\
\hline 2 & 0.8 & 0.893981 & 0.467399 & 0.313561 & 0.204297 & $0.94117(0.68750)$ & $0.947891(0.02226)$ & 0.94768 \\
\hline 3 & 0.4 & 5.074636 & 1.971962 & 0.281418 & 0.149677 & $0.85275(0.65326)$ & $0.858566(0.02516)$ & 0.85835 \\
\hline 4 & 0.3 & 5.371626 & 1.995820 & 0.272313 & 0.133708 & $0.90371(0.32569)$ & $0.906268(0.04324)$ & 0.90666 \\
\hline 1 & 1.1 & 0.333861 & 0.131914 & 0.333105 & 0.129923 & $1.05601(0.50254)$ & $1.060826(0.04843)$ & 1.06134 \\
\hline 2 & 0.6 & 1.512930 & 0.802700 & 0.379417 & 0.250283 & $0.92528(0.59649)$ & $0.930113(0.07810)$ & 0.93084 \\
\hline 3 & 0.4 & 4.278991 & 1.715424 & 0.318201 & 0.173313 & $0.87526(0.53749)$ & $0.879977(0.00147)$ & 0.87999 \\
\hline 4 & 0.3 & 10.015007 & 3.820495 & 0.297109 & 0.149231 & $0.83552(0.79887)$ & $0.841654(0.06958)$ & 0.84224 \\
\hline 1 & 0.9 & 0.327231 & 0.127022 & 0.333105 & 0.129923 & $1.03871(0.31394)$ & $1.040568(0.13551)$ & 1.04198 \\
\hline 2 & 0.7 & 1.356964 & 0.696043 & 0.379417 & 0.250283 & $0.91850(0.74083)$ & $0.925756(0.04388)$ & 0.92535 \\
\hline 3 & 0.4 & 4.233389 & 1.657464 & 0.318201 & 0.173313 & $0.87662(0.52277)$ & $0.881255(0.00284)$ & 0.88123 \\
\hline 4 & 0.1 & 11.178563 & 4.200250 & 0.297109 & 0.149231 & $0.97418(0.14969)$ & $0.974368(0.13038)$ & 0.97564 \\
\hline
\end{tabular}

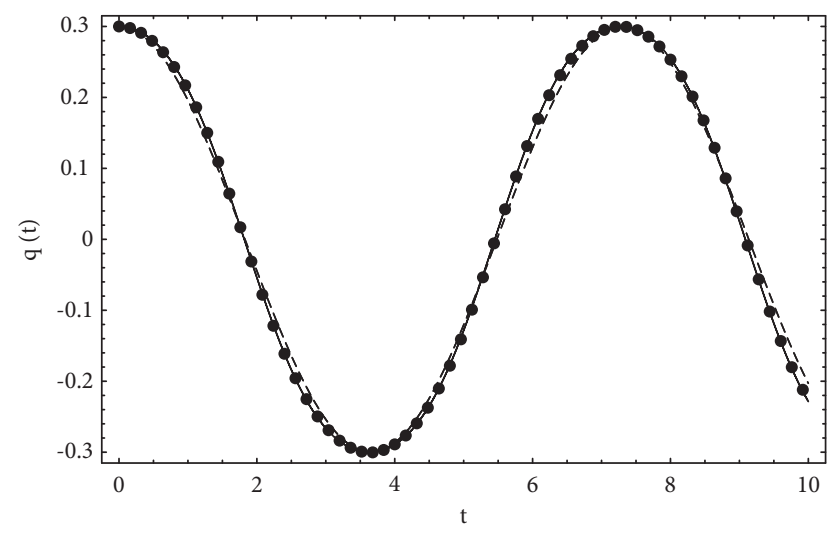

Figure 2: Comparison between approximate and exact solutions under the parameter values (46); •... exact solution; --- zeroth-order approximation; _ first-order approximation.

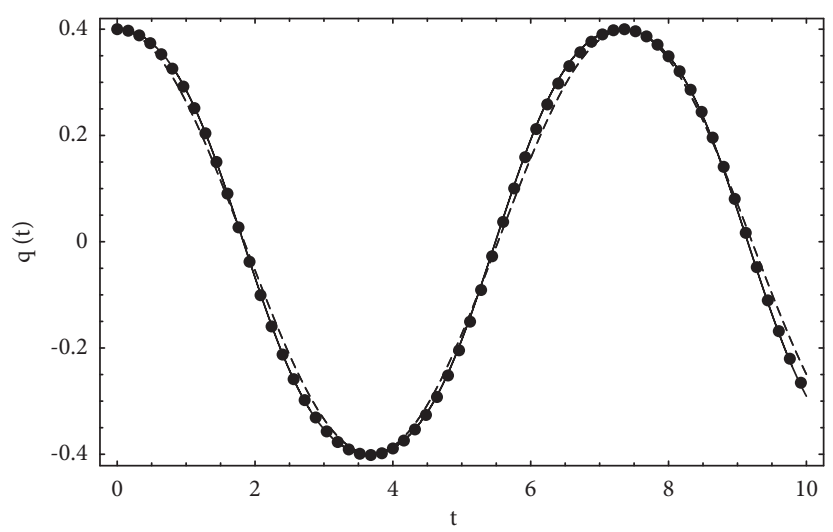

FIGURE 3: Comparison between approximate and exact solutions under the parameter values (47); •... exact solution; --- zeroth-order approximation; - first-order approximation. 


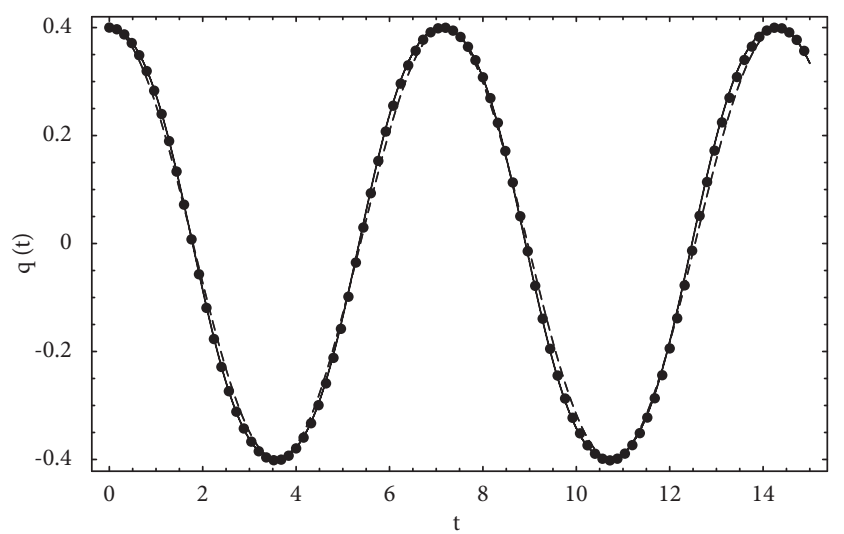

Figure 4: Comparison between approximate and exact solutions under the parameter values $(48) ; \cdots$.. exact solution; --- zerothorder approximation; — first-order approximation.

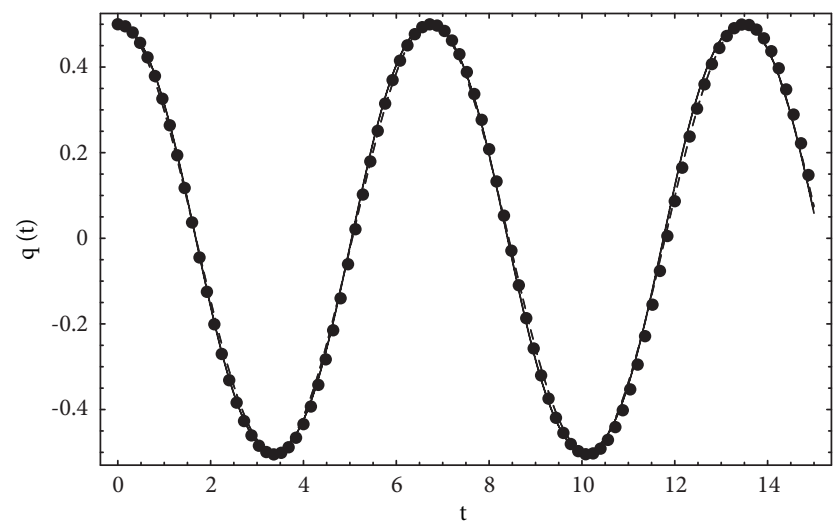

FIgURE 5: Comparison between approximate and exact solutions under the parameter values (49); .... exact solution; --- zerothorder approximation; _ f first-order approximation.

it is noteworthy that the first-order approximate solutions are almost similar to the exact solutions. This shows that the accuracy of the results obtained by this method is very high.

\section{Conclusions}

The global residue harmonic balance method has been employed to obtain the approximate periodic solution and frequency for a well-known system of strongly nonlinear oscillators in engineering, namely, the large amplitude free vibration of a restrained uniform beam carrying an intermediate lumped mass.

(i) The brief descriptions of the beam and the basic idea of the GRHBM are introduced.

(ii) The GRHBM can provide a high accuracy by considering the residual errors in the process of each order approximation. The solution procedure of the GRHBM is simple and clear. The higher-order approximate solution and frequency for the considered problem by GRHBM can be obtained easily. (iii) By comparing with the previously existing results and the exact solutions, it is shown that the GRHBM is an accurate, convenient, and efficient method.

(iv) The GRHBM can be easily extended to other nonlinear oscillators in engineering and physics.

\section{Data Availability}

The data used to support this study are available from the corresponding author upon request.

\section{Conflicts of Interest}

The authors declare that there are no conflicts of interest regarding the publication of this paper.

\section{Acknowledgments}

This paper was supported by the Research Fund Projects of Zhoukou Normal University (ZKNUC2016012), the Key Teachers Research Foundation of the Henan Higher Education Institutions of China (2019GGJS217 and 2020GGJS217), the Research Foundation of the Henan Higher Education Institutions of China (21A110027), and the Key Teachers Research Foundation of Zhoukou Normal University.

\section{References}

[1] A. H. Nayfeh, Introduction to Perturbation Methods, Wiley, Hoboken, NY, USA, 1981.

[2] J. K. Kevorkian and J. D. Cole, Perturbation Methods in Applied Mathematics, Springer-Verlag, Berlin, Germany, 1981.

[3] F. Verhulst, Methods and Applications of Singular Perturbations: Boundary Layers and Multiple Timescales Dynamics, Springer-Verlag, Berlin, Germany, 2005.

[4] J. H. He, "Some asymptotic methods for strongly nonlinear equations," International Journal of Modern Physics B, vol. 20, no. 10, pp. 1141-1199, 2016.

[5] Y. S. Yun and C. Temuer, "Application of the homotopy perturbation method for the large deflection problem of a circular plate," Applied Mathematical Modelling, vol. 39, no. 3-4, pp. 1308-1316, 2015.

[6] A. I. Enagi, M. Bawa, and A. M. Sani, "Mathematical study of diabetes and its complication using the homotopy perturbation method," International Journal of Mathematics and Computer Science, vol. 12, no. 1, pp. 43-63, 2017.

[7] I. A. Tijani, Y. F. Wu, and C. W. Lim, "Energy balance method for modeling ultimate strain of fiber-reinforced polymerrepaired concrete," Structural Concrete, vol. 21, no. 2, pp. 1-17, 2019.

[8] G. Liu, Z. R. Lv, J. K. Liu, and Y. M. Chen, "Quasi-periodic aeroelastic response analysis of an airfoil with external store by incremental harmonic balance method," International Journal of Non Linear Mechanics, vol. 100, pp. 10-19, 2018.

[9] Y. D. Li and Y. R. Yang, "Vibration analysis of conveying fluid pipe via he's variational iteration method," Applied Mathematical Modelling, vol. 43, pp. 409-420, 2017.

[10] M. M. Peiravi and J. Alinejad, "Hybrid conduction, convection and radiation heat transfer simulation in a channel with 
rectangular cylinder," Journal of Thermal Analysis and Calorimetry, vol. 140, no. 6, pp. 2733-2747, 2020.

[11] M. Ho, S. Leclaire, M. Reggio, and J. Y. Trepanier, "Stochastic effects of $2 \mathrm{D}$ random arrays of cylinders on rarefied gas permeability using the lattice boltzmann method," Transport in Porous Media, vol. 136, no. 2, pp. 1-31, 2021.

[12] R. Bahoosh, R. Khalili, R. Amin, and A. Noghrehabadi, "An axisymmetric lattice boltzmann method simulation of forced convection heat transfer for water/aluminum oxide nanofluid through a tube under constant heat flux on wall," International Journal of Heat and Mass Transfer, vol. 8, no. 8, pp. 71-85, 2021.

[13] F. H. Easif, S. A. Manaa, and A. J. Sabali, "Modified variational iteration and homotopy analysis method for solving variable coefficient variant boussinesq system," General Letters in Mathematics, vol. 8, no. 1, pp. 26-32, 2020.

[14] M. Vakilzadeh, R. Vatankhah, and M. Eghtesad, "Investigation of dynamic pull-in instability of suspended microchannel resonators using homotopy analysis method," Journal of the Brazilian Society of Mechanical Sciences and Engineering, vol. 43, no. 6 , pp. 1-9, 2021.

[15] S. Suharsono, S. Wulandari, A. Nuryaman, M. Usman, W. Wamiliana, and J. Daoud, "Solution of the reverse flow reactor model using homotopy analysis method," IIUM Engineering Journal, vol. 22, no. 1, pp. 129-137, 2020.

[16] K. A. Abed, "Solving Kuramoto-Sivashinsky equation by the new iterative method and estimate the optimal parameters by using PSO algorithm," TELKOMNIKA Indonesian Journal of Electrical Engineering, vol. 19, no. 2, pp. 709-714, 2020.

[17] R. A. Talarposhti, Z. Asadi, H. Rezazadeh, D. D. Ganji, and A. Bekir, "Analytical solution for differential nonlinear and coupled equations in micropolar nanofluid flow between rotating parallel plates," The European Physical Journal Special Topics, vol. 228, no. 12, pp. 2601-2617, 2019.

[18] M. B. Gerdroodbary, "Application of neural network on heat transfer enhancement of magnetohydrodynamic nanofluid," Heat Transfer, vol. 49, no. 1, pp. 197-212, 2020.

[19] A. Khalouta and A. Kadem, "A new modification of the reduced differential transform method for nonlinear fractional partial differential equations," Journal of Applied Mathematics and Computational Mechanics, vol. 19, no. 3, pp. 45-58, 2020.

[20] M. Ramos, "One-step hybrid block method containing third derivatives and improving strategies for solving bratu's and troesch's Problems," Numerical Mathematics Theory Methods and Applications, vol. 13, no. 4, pp. 946-972, 2020.

[21] M. M. Peiravi and J. Alinejad, "Numerical analysis of secondary droplets characteristics due to drop impacting on $3 \mathrm{D}$ cylinders considering dynamic contact angle," Meccanica, vol. 55, no. 10, pp. 1975-2002, 2020.

[22] P. Ju and X. Xue, "Global residue harmonic balance method to periodic solutions of a class of strongly nonlinear oscillators," Applied Mathematical Modelling, vol. 38, no. 24, pp. 6144-6152, 2014.

[23] P. Ju, "Global residue harmonic balance method for a nonlinear oscillator with discontinuity," Applied Mathematical Modelling, vol. 39, no. 21, pp. 6738-6742, 2015.

[24] P. Ju, "Global residue harmonic balance method for helmholtz-duffing oscillator," Applied Mathematical Modelling, vol. 39, no. 8, pp. 2172-2179, 2015.

[25] M. Mohammadian and M. Shariati, "Approximate analytical solutions to a conservative oscillator using global residue harmonic balance method," Chinese Journal of Physics, vol. 55, no. 1, pp. 47-58, 2016.
[26] G. Ismail and M. A. Hosen, "Global residue harmonic balance method for obtaining higher-order accurate solutions to the strongly nonlinear oscillator," Thai Journal of Mathematics, vol. 18, no. 4, pp. 1947-1959, 2020.

[27] M. N. Hamdan and N. H. Shabaneh, "On the large amplitude free vibrations of a restrained uniform beam carrying an intermediate lumped mass," Journal of Sound and Vibration, vol. 199, no. 5, pp. 711-736, 1997.

[28] S. S. Ganji, D. D. Ganji, M. G. Sfahani, and S. Karimpour, "Application of AFF and HPM to the systems of strongly nonlinear oscillation," Current Applied Physics, vol. 10, no. 5, pp. 1317-1325, 2010. 